\title{
Observation of field-induced domain wall propagation in magnetic nanowires by magnetic transmission X-ray microscopy
}

\author{
M. T. Bryan ${ }^{1}$, P. W. Fry ${ }^{2}$, P. J. Fischer ${ }^{3}$, and D. A. Allwood ${ }^{1 *}$ \\ ${ }^{1}$ Department of Engineering Materials, University of Sheffield, Sheffield S1 3JD, UK \\ ${ }^{2}$ Centre for Nanoscience and Technology, University of Sheffield, Sheffield S3 7HQ, UK \\ ${ }^{3}$ LBNL/CXRO, 1 Cyclotron Road, Berkeley, California 94720, USA
}

\begin{abstract}
Magnetic transmission X-ray microscopy (M-TXM) is used to image domain walls in magnetic ring structures formed by a $300 \mathrm{~nm}$ wide, $24 \mathrm{~nm}$ thick $\mathrm{Ni}_{81} \mathrm{Fe}_{19}$ nanowire. Both transverse and vortex type domain walls are observed after application of different field sequences. Domain walls can be observed by comparing images obtained from opposite field sequences, or else domain wall propagation observed by comparing successive images in a particular field sequence. This demonstrates the potential use of M-TXM in developing and understanding planar magnetic nanowire behavior.
\end{abstract}

*E-mail: d.allwood@ sheffield.ac.uk 


\section{Introduction}

The geometry of planar magnetic nanowires creates a simple magnetic structure in which domain walls across the wire width separate domains oriented along the wire length. Domain walls can be propagated using externally applied fields ${ }^{1,2}$ or electrical current in the nanowires. ${ }^{3-}$

${ }^{7}$ Furthermore, domain walls can be positioned using geometrical features, e.g. corners ${ }^{1,6,8,9}$ or notches, and interact at wire junctions. ${ }^{8,9}$ This has led to several proposals for using domain walls in nanowire networks for memory, ${ }^{10,11}$ logic, ${ }^{8,9}$ and sensor ${ }^{12}$ applications.

There is now a great deal of interest in understanding the nature of domain wall propagation in nanowires and domain wall interactions with nanowire features. Several techniques assist in this by allowing the nanowire magnetization to be imaged. Magnetic force microscopy (MFM) has been used extensively to image the stray field from domain walls in nanowires, ${ }^{6,7,13}$ although great care has to be taken to avoid altering the magnetic state of the nanowires with the magnetic tip used in MFM. Scanning electron microscopy with polarization analysis $(\text { SEMPA })^{14}$ and photoelectron emission microscopy (PEEM) ${ }^{15}$ provide excellent sensitivity to surface magnetization but samples must be in vacuum and SEMPA cannot image surfaces when magnetic fields are applied to the samples. Lorentz-transmission electron microscopy, ${ }^{16,17}$ also performed in vacuum, requires samples to be on electron-transparent substrates and of a minimum thickness (typically $20 \mathrm{~nm}$ for $\mathrm{Ni}_{81} \mathrm{Fe}_{19}$ ) but nevertheless can produce excellent images of domain wall structure in magnetic nanowires. ${ }^{17}$ Finally, magnetic transmission X-ray microscopy (M-TXM) ${ }^{18,19}$ can produce in-field magnetization images from samples in air, although X-ray-transparent substrates (similar to those in Lorentz TEM) must again be used. Magnetic contrast is obtained by X-ray magnetic circular dichroism since the transmission of circularly polarized X-rays with an energy close to resonant absorption edges depends strongly on the relative orientation between the projection of the sample's magnetization onto the photon propagation direction. 
M-TXM has been used to image magnetization processes in isolated elements. ${ }^{18}$ Recently, MTXM was used to observe current-driven propagation of a 'vortex' type domain wall in $1 \mu \mathrm{m}$ wide, $80 \mathrm{~nm}$ thick nanowires. ${ }^{20}$ Here, we show how M-TXM can be used to observe domain walls with vortex or transverse structure propagating in nanowires with dimensions closer to those used in proposed applications.

\section{Experimental}

Nanowires were patterned on $100 \mathrm{~nm}$ thick $\mathrm{Si}_{3} \mathrm{~N}_{4}$ membranes by standard electron beam lithography and lift-off techniques, although ultra-sonic treatment was avoided to prevent damage to the membrane. The samples were metalized by thermal evaporation of $24 \mathrm{~nm}$ $\mathrm{Ni}_{81} \mathrm{Fe}_{19}$ using a custom-built evaporator. The sample design consisted of a $4 \mu \mathrm{m}$ diameter ring with a straight wire connected radially to the outer edge of the ring. The straight wire terminates in a $180^{\circ}$ bend but is not significant for the experiments discussed here. The wire width of the circle is $300 \mathrm{~nm}$ while the straight wire and $180^{\circ}$ bend are $200 \mathrm{~nm}$ wide. Figure 1 shows a scanning electron micrograph of a complete structure, illustrating the ring, straight wire and bend. Figure 1 also defines the $x$ - and $y$-directions, along which the magnetic fields $H_{x}$ and $H_{y}$ were applied, respectively.

M-TXM was performed at beamline 6.1.2 operated by the Center for X-ray Optics at the Advanced Light Source, in Berkeley $\mathrm{CA}^{21}$ using the optical configuration consisting of Fresnel zone plates (Fig. 2), which is described in detail elsewhere. ${ }^{22}$ A condenser zone plate (CZP) provides a hollow cone illumination of the specimen with circularly polarized X-rays being

selected by a movable aperture to pick off-orbit bending magnet radiation. Together with a 12 $\mu \mathrm{m}$ diameter pinhole the $\mathrm{CZP}$ acts as linear monochromator so that $\mathrm{X}$-rays with an energy corresponding to the L-edges of either $\mathrm{Ni}(854 \mathrm{eV})$ or $\mathrm{Fe}(706 \mathrm{eV})$ can be selected. Magnetic fields pointing in two orthogonal directions to the photon propagation direction can be applied 
to the samples, which were mounted at a $30^{\circ}$ tilt angle to provide sensitivity to in-plane magnetization. A high resolution micro zone plate was used to image the sample's magnetization projection onto a CCD camera. The images were recorded under ambient conditions and at various externally applied magnetic fields. In order to increase the magnetic contrast and to remove non-magnetic background contributions, difference images were created from images obtained under different applied magnetic fields but identical X-ray polarization to observe changes in magnetization. The two component images represent the initial (before field change) and final (after field change) magnetization states. Here, this procedure provides light (dark) contrast for positive (negative) magnetization changes in the $x$-direction. The maximum fields applied to samples were $H_{x}= \pm 500$ Oe and $H_{y}= \pm 100 \mathrm{Oe}$.

\section{Results and Discussion}

Initially, we observed static domain walls in the nanowire ring by applying magnetic fields along one axis only. Figure 3(a) is obtained from images at remanence following $H_{x}=+500 \mathrm{Oe}$ (initial) and remanence following $H_{x}=-500$ Oe (final). Strong contrast is observed at the upper and lower sections of the ring due to magnetization reversal. The intensity contrast between the two remanent images at the top of the ring structures is $10 \%$. Less contrast is observed in the adjacent ring side sections. This is due to magnetization lying approximately parallel to the ring

edges and, consequently, having very little component in the $x$-direction. Interestingly, there is also a dark structure on the far right of the ring that shows a transverse domain wall structure of width $\sim 90 \mathrm{~nm}$. The dark contrast indicates that, in this case, the domain walls at remanence have magnetization parallel to the previous applied field direction. However, other images indicate that this is not always the case. This can be expected since 500 Oe is below the local anisotropy field of the wire structure, so saturation will not be achieved.

Figure 3(b) shows an image obtained from the conditions of remanence after $H_{x}=+500 \mathrm{Oe}$ (initial) and during $H_{x}=-500$ Oe (final). Surprisingly, the domain wall contrast is reduced and 
appears to be split. This type of image was highly repeatable. Our explanation for this is that the domain wall relaxes to different positions at remanence and when $\left|H_{x}\right|=500$ Oe. This could be due to a defect in the wire introducing position-dependent potentials on the domain wall position. Of the two domain wall positions in Fig. 3(b), the lower position corresponds to the domain wall location in Fig. 3(a) while the upper position is reached when $H_{x}=500$ Oe.

Figure 4 shows a sequence of domain wall images that were obtained by applying fields $H_{x}$ and $H_{y}$. Initially, fields $H_{x}=500 \mathrm{Oe}, H_{y}=0$ Oe were applied and then removed to prepare a transverse domain wall on the right hand side of the ring, as in Fig. 3. Image $I_{l}$ recorded this state. Fields $H_{x}=0 \mathrm{Oe}, H_{y}=30$ Oe were then applied and image $I_{2}$ collected. Figure 4(a) shows an image obtained using $I_{1}$ and $I_{2}$ as the initial and final states, respectively. The separated light and dark contrast regions on the right hand side of the ring indicate that a transverse domain wall has propagated $250 \mathrm{~nm}$ in the ring wire between the initial and final positions $P_{1}$ and $P_{2}$, respectively. The magnetization configuration of the initial and final states is shown schematically in Fig. 4(b) and (c). As an aid to understanding Fig. 4(a), the schematic diagrams show significant magnetization changes in black and unchanged magnetization in gray. Unlike in Fig. 3, the initial domain wall magnetization in Fig. 4(a) is opposite to the $+x$ initialization field direction.

The applied fields were then changed to $H_{x}=-100 \mathrm{Oe}, H_{y}=30$ Oe and a new image $I_{3}$ recorded. Figure 4(d) shows an image that uses $I_{2}$ as the new initial state and $I_{3}$ as the new final state. The magnetic configurations of the initial and final states are illustrated schematically in Fig. 4(e) and (f). The initial position $P_{1}$ of the transverse domain wall is again indicated by the light contrast region in Fig. 4(d). The dark track above $P_{l}$ indicates that the wall has propagated to position $P_{2}$ right at the top of the ring. The dark contrast here is now due to a reversal in magnetization parallel to the nanowire sides rather than imaging the domain wall itself. However, the domain wall structure is still evident at the end of the dark streak at $P_{2}$. The dark contrast at $P_{2}$ only covers half of the nanowire width, indicating that the domain wall has 
transformed into a vortex structure. This is understood by viewing the change in magnetization (black lines) shown in Fig. 4(f); the lower half of the vortex domain wall structure has magnetization parallel to the magnetic domain at this position in the initial state. Previous modelling $^{23}$ of field-driven domain wall propagation in wires with a cross-sectional area $\sim 40 \%$ of those used here predicted a transformation from a vortex to transverse domain wall configuration. The larger cross-sectional area used here is probably responsible for the opposite transformation shown in Fig. 4(d).

The applied field was now changed again to $H_{x}=100 \mathrm{Oe}, H_{y}=0$ Oe and image $I_{4}$ obtained. The image in Fig. 4(g) results from $I_{3}$ and $I_{4}$ being used as the initial and final states, respectively, with the two configurations being illustrated in Fig. 4(h) and (i). The light contrast regions at $P_{I}$ and $P_{2}$ indicate the initial and final positions of the domain wall, this time propagating $\sim 250 \mathrm{~nm}$ back towards its original location in Fig. 4(a). The light contrast regions again extend only halfway across the nanowire, although on opposite sides of the wire at $P_{1}$ and $P_{2}$. This is explained by the propagating vortex domain wall structure illustrated in Fig. 4(h) and (i) and confirms that Fig. 4(d) shows a vortex wall structure in the final state. The vortex walls in Fig. 4(g) are $270 \mathrm{~nm}$ wide, substantially wider than transverse walls in the same ring structure.

\section{Conclusion}

We have shown that transverse and vortex domain walls can be observed in $300 \mathrm{~nm}$ wide $\mathrm{Ni}_{81} \mathrm{Fe}_{19}$ nanowires using magnetic X-ray transmission (M-TXM) microscopy. Domain wall propagation is easily identified either using domain or domain wall magnetization, depending on the local angle between the wire and the microscope's sensitivity direction. We have also observed a transition in domain wall structure from transverse to vortex. This is significant for domain wall technologies that are attempting to control the position of domain walls using geometrical features in nanowires since the operation of these is often critically dependent upon domain wall structure. 


\section{Acknowledgements}

MTB, PWF and DAA thank EPSRC for financial support, including grants GR/T02959/01 and EP/D056683/1. This work was supported by the Director, Office of Science, Office of Basic Energy Sciences, Materials Sciences and Engineering Division, of the U.S. Department of Energy under Contract No. DE-AC02-05CH11232.

\section{References}

1 D. Atkinson, D. A. Allwood, G. Xiong, M. D. Cooke, C. C. Faulkner, and R. P. Cowburn, Nature Mater. 2, 85 (2003).

2 G. S. D. Beach, C. Nistor, C. Knutson, M. Tsoi, and J. L. Erskine, Nature Mater. 4, 741 (2005).

3 N. Vernier, D. A. Allwood, D. Atkinson, M. D. Cooke, and R. P. Cowburn, Europhys. Lett. 65, 526 (2004).

4 J. Grollier, P. Boulenc, V. Cros, A. Hamzic, A. Vaurès, A. Fert, and G. Faini, Appl. Phys. Lett. 83, 509 (2003).

5 M. Hayashi, L. Thomas, C. Rettner, R. Moriya, Y. B. Bazaliy, and S. S. P. Parkin, Phys. Rev. Lett. 98, 037204 (2007).

6 L. Thomas, M. Hayashi, X. Jiang, R. Moriya, C. Rettner, and S. S. P. Parkin, Nature 443, 197 (2006).

7 A. Yamaguchi, T. Ono, S. Nasu, K. Miyake, K. Mibu, and T. Shinjo, Phys. Rev. Lett. 92, 077205 (2003).

8 D. A. Allwood, G. Xiong, and R. P. Cowburn, J. Appl. Phys. 100, 123908 (2006). 
D. A. Allwood, G. Xiong, C. C. Faulkner, D. Atkinson, D. Petit, and R. P. Cowburn, Science 309, 1688 (2005). S. S. P. Parkin, U.S. Patent 20040252539 (2004).

R. D. McMichael, J. Eicke, M. J. Donahue, and D. G. Porter, J. Appl. Phys. 87, 7058 (2000).

M. Diegel, R. Mattheis, and E. Halder, IEEE Trans. Magn. 40, 2655 (2004). X. Zhu, D. A. Allwood, G. Xiong, R. P. Cowburn, and P. Grütter, Appl. Phys. Lett. 87, 062503 (2005).

F. C. S. da Silva, W. C. Uhlig, A. B. Kos, S. Schima, J. Aumentado, J. Unguris, and D. P. Pappas, Appl. Phys. Lett. 85, 6022 (2004).

15 M. Kläui, M. Laufenberg, L. Heyne, D. Backes, U. Rüdiger, C. A. F. Vaz, J. A. C. Bland, L. J. Heyderman, S. Cherifi, A. Locatelli, T. O. Mentes, and L. Aballe, Appl. Phys. Lett. 88, 232507 (2006). J. N. Chapman and M. R. Scheinfein, J. Magn. Magn. Mater. 200, 729 (1999). C. Brownlie, S. McVitie, J. N. Chapman, and C. D. W. Wilkinson, J. Appl. Phys. 100, 033902 (2006). P. Fischer, Current Opinion in Solid State and Materials Science 7, 173 (2003). J. B. Kortright, D. D. Awschalom, J. Stoehr, S. D. Bader, Y. U. Idzerda, S. S. P. Parkin, I. K. Schuller, and H. C. Siegmann, J. Magn. Magn. Mater. 207, 7 (1999). G. Meier, M. Bolte, R. Eiselt, B. Krüger, D. H. Kim, and P. Fischer, Phys. Rev. Lett. 98, 187202 (2007). www-cxro.lbl.gov. P. Fischer, D. H. Kim, W. Chao, J. A. Liddle, E. H. Anderson, and D. T. Attwood, Materials Today 9, 26 (2006). 

(2005). 


\section{Figure Captions}

Figure 1 Scanning electron micrograph of the $24 \mathrm{~nm}$ thick $\mathrm{Ni}_{81} \mathrm{Fe}_{19}$ structure on a $\mathrm{Si} \mathrm{N}$ membrane. Also shown are the orthogonal $x$ - and $y$-directions.

Figure 2 Schematic of optical arrangement for performing magnetic transmission X-ray microscopy at beamline 6.1.2 at the Advanced Light Source, Berkeley, CA.

Figure 3 Difference magnetic transmission X-ray microscopy images showing magnetization changes in the $\mathrm{Ni}_{81} \mathrm{Fe}_{19}$ ring and direction of magnetic field $H_{x}$. The images are obtained from conditions (a) remanence after $H_{x}=+500$ Oe (initial) and remanence after $H_{x}=-500 \mathrm{Oe}$ (final), and (b) remanence after $H_{x}=+500 \mathrm{Oe}$ (initial) and $H_{x}=-500 \mathrm{Oe}$ (final). The insets show magnified and higher contrast images of the domain wall regions.

Figure 4 Difference magnetic transmission X-ray microscopy images and schematic diagrams of domain walls in the initial $\left(P_{1}\right)$ and final $\left(P_{2}\right)$ positions for the difference image. Successive images result from applying new field conditions compared with the previous image. Initial fields of $H_{x}=H_{y}=0$ Oe after $H_{x}=500$ Oe, $H_{y}=0$ Oe were followed by (a) - (c) $H_{x}=0$ Oe, $H_{y}=30$ Oe, (d) - (f) $H_{x}=-$ $100 \mathrm{Oe}, H_{y}=30 \mathrm{Oe},(\mathrm{g})-(\mathrm{i}) H_{x}=100 \mathrm{Oe}, H_{y}=0 \mathrm{Oe}$. Also indicated are the orthogonal directions in which $H_{x}$ and $H_{y}$ were applied. 


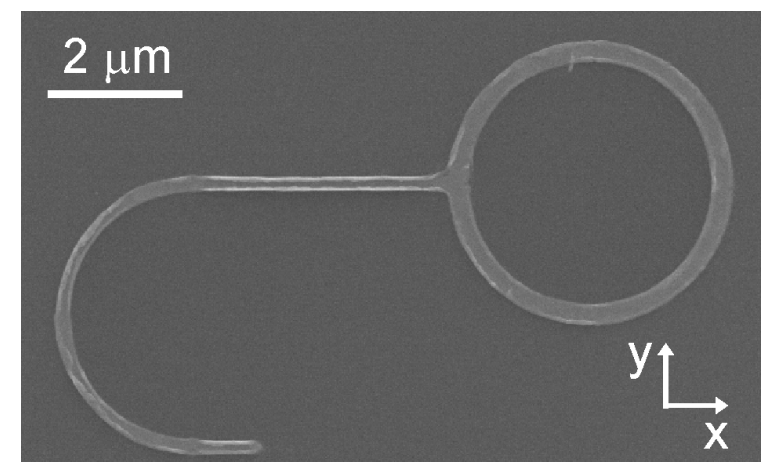

Figure 1 


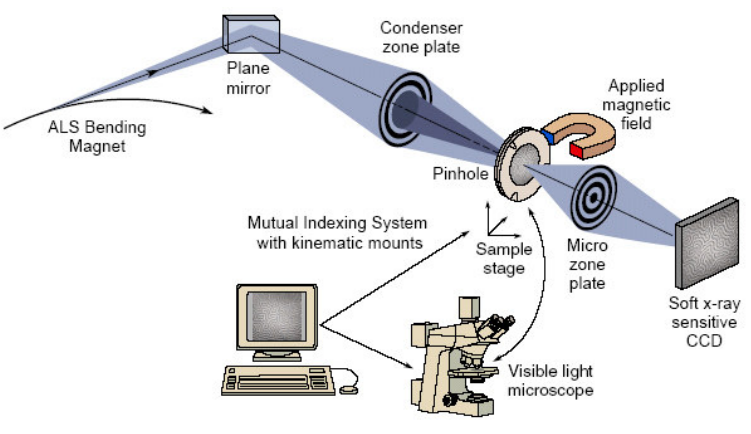

Figure 2 

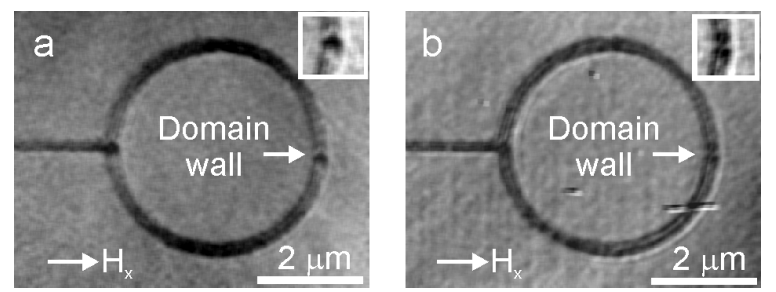

Figure 3 

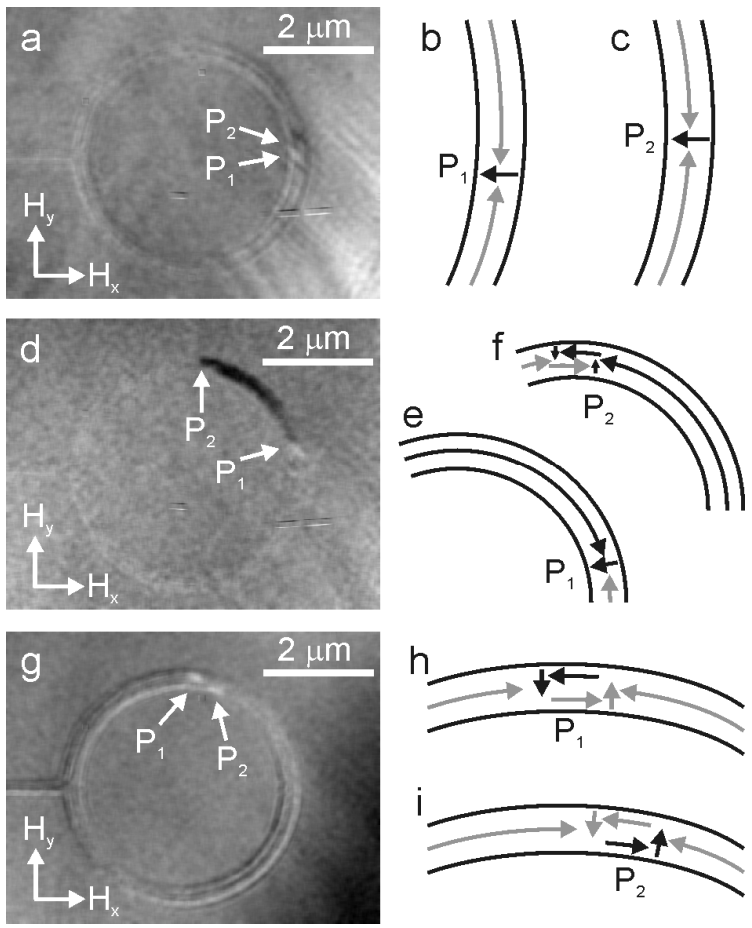

Figure 4 